\title{
Images of the month 2: A target sign in COVID-19 pneumonia
}

\author{
Authors: Jitender Singh ${ }^{A}$ and Tarika Sharma ${ }^{B}$
}
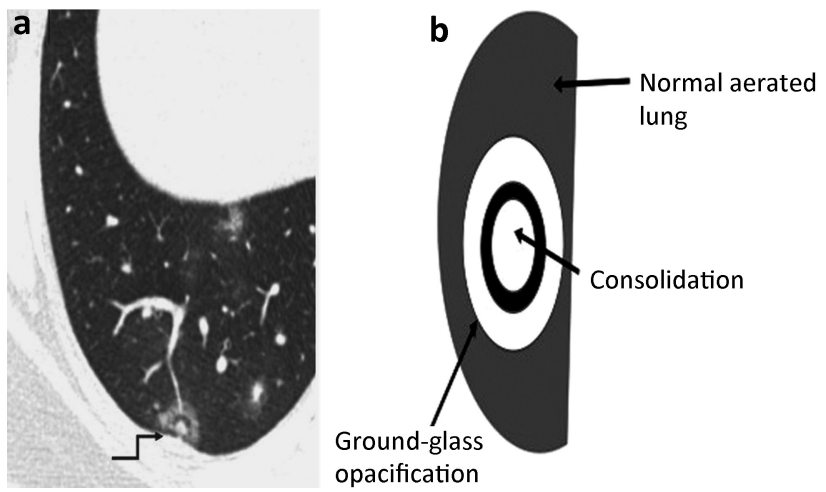

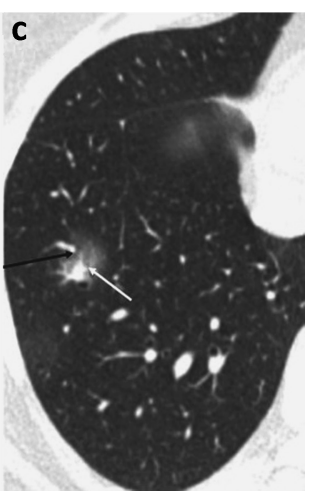

Fig 1. Target sign and halo sign. a) Computed tomography (coned down axial section) of the right lung showing an irregular ring-like opacity containing in its centre, a small nodular ground-glass opacification surrounding a very small vessel (arrow); also of note are small patchy ground-glass opacities. b) Graphic showing how to identify a target sign. c) Computed tomography (coned down axial section at cardiac level) showing combination of central nodular opacity and the peripheral irregular rim of groundglass opacification; also of note are small patchy ground-glass opacities.
A target sign has not been described in other viral or bacterial pneumonia on computed tomography of the chest in literature. It could represent a hallmark of COVID-19 pneumonia, given the good correlating clinical context and prevalence of COVID-19.

KEYWORDS: computed tomography, reverse transcription polymerase chain reaction, COVID-19, ground-glass opacifications, target sign

DOI: $10.7861 /$ clinmed.2021-0377

\section{Case presentation}

A 37-year-old man presented with fever of 5 days duration. At the time of admission, the patient had breathlessness along with hypoxia, reverse transcription polymerase chain reaction was positive. X-ray of the chest revealed small patchy ground-glass haziness in bilateral lower lung fields (right>left). The patient was shifted to the high dependency unit due to worsening of respiratory symptoms on day 9 and a repeat computed tomography (CT) was performed which showed consolidation

Authors: ${ }^{\text {A }}$ consultant, Shanti Mukund Hospital, New Delhi, India; ${ }^{B}$ assistant professor, College of Nursing at the Institute of Liver and Biliary Sciences, New Delhi, India and ground-glass opacification (GGO) along with a target sign in subpleural location of right lower lung lobe (Fig 1a). He was managed with oxygen therapy and other medications. The patient improved and was discharged in a stable condition on day 17.

\section{Discussion}

The sign was first described in June 2020 in COVID-19 pneumonia on CT of the chest. ${ }^{1}$

The sign has been depicted as a central high attenuation focus surrounded by one or more dense complete or incomplete ring-like consolidations, forming one or more circles on CT of the chest in patients with COVID-19 pneumonia (Fig 1b). ${ }^{1}$ The pathophysiological sign is a manifestation of organising pneumonia (OP) which represents peripheral opacities whereas the central nodular opacity represents the perivascular inflammation or focal enlargement of the pulmonary artery (vessel sign). ${ }^{2,3}$ The pulmonary target sign usually coexists with other typical OP features including the reverse halo or halo sign which are characterised by peripheral consolidation with central GGO and central consolidation with peripheral GGO, respectively (Fig 1c). ${ }^{4}$ Further patho-radiological studies are required to investigate the value of this sign in the specificity with COVID-19 pneumonia.

\section{References}

1 Müller CIS, Müller NL. Chest CT target sign in a couple with COVID-19 pneumonia. Radiol Bras 2020;53:252-4. 
2 Pogatchnik BP, Swenson KE, Sharifi H et al. Radiology-pathology correlation in recovered COVID-19, demonstrating organizing pneumonia. Am J Respir Crit Care Med 2020;202:598-9.

3 Saburi A, Schoepf UJ, Ulversoy KA et al. From radiological manifestations to pulmonary pathogenesis of COVID-19: a bench to bedside review. Radiol Res Pract 2020;2020:8825761.

4 Lang M, Som A, Carey D et al. Pulmonary vascular manifestations of COVID-19 pneumonia. Radiol Cardiothorac Imaging 2020;2:e200277.
Address for correspondence: Dr Jitender Singh, Department of Intervention Radiology Shanti Mukund Hospital, 2 Institutional Area, Bhartendu Harish Chandra Marg, Vikas Marg Extension, Karkardooma, New Delhi, Delhi 110092, India.

Email: introductory2008@gmail.com 